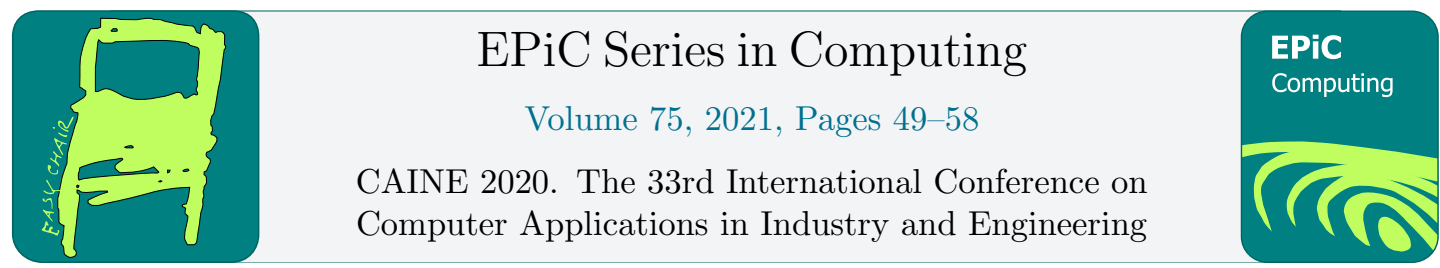

\title{
Salient Benefits of Platooning
}

\author{
Henry Hexmoor and Reem Saad Alshiddi \\ School of Computing at Southern Illinois University, Carbondale, IL, USA \\ hexmoor@cs.siu.edu, rema.shiddi@siu.edu
}

\begin{abstract}
This paper offers insights about the most important properties of vehicular platooning. A key feature is determining circumstances when platooning will reduce travel times and emission reductions. In each roadmap, we highlight quantifiable measures corresponding to travel times corresponding to optimal platooning locations. We have performed simulated experiments that showed using platooning provides nontrivial financial benefits for organizations deploying platoons as well as overall travel time reductions.
\end{abstract}

Keywords- platoon, platooning point, heavy-duty vehicle, arrival time, waiting time.

\section{Introduction}

Platooning is a solution that can achieve salient road use efficiency goals [1]. In this paper, we will focus on truck platooning in specific that are used for transporting freight and how truck platooning can facilitate freight movement with several benefits including increased road capacity and reducing fuel consumption [2] [3].

Experiments described in [4] shows that the following truck driving at a $10 \mathrm{~m}$ distance could save fuel up to $20 \%$ comparing to isolated driving. Meanwhile, the leading truck experienced reduction on fuel consumption as well. It is known that vehicle $\mathrm{CO}_{2}$ emission is directly proportional to the fuel consumption [5]. The results could be higher with a shorter inter-vehicle distance and be in a platoon for longer time.

Speaking of truck platooning, some research results defined that truck platooning can be represented in making the trucks align into a group whereas a distance between them be reduced as could be in a convoy formation at highway speeds. Each platoon contains two types of trucks: the lead truck (LT) and the following truck (FT) [6]. The lead truck is the first truck in a platoon, and it leads the other trucks on roads. The following trucks are the trucks that are following the lead truck in its speed and movements. Engineers have found ways to maintain short distances between trucks in a platoon that could improve aerodynamic drag. The lead truck stays responsible for traditional acceleration and braking, while all what following trucks should do is following the leader. It is worth mentioning that be better to identify the leader and the follower trucks regarding to what was mention to vehicle platooning policy from department of transportation in Pennsylvania [7]. 
Some researches named the trucks that are responsible of freight delivery as Heavy-Duty Vehicles (HDV). Commonly, the main factor of HDV delivery is the arrival time. The arrival time basically depends on many other factors such as vehicle speed, road obstacles, weather conditions, traffic, ..., etc. In the same context, delivery economic is matter for delivery companies' side in many aspects that should be considered like total cost, travel time, waiting time, customer service level fuel cost [4]. Therefore, congestion and long travel time will cause addition cost for fuel, and negatively extra emission for the environment. Moreover, longer journeys are not preferred to drivers, and they may cause some accidents due to the exhaustion.

Generally, we target how using truck platooning can alter on roads efficiency, and how companies that are providing the trucks can get benefits from platooning. In addition, if some trucks are needed to be platooned, where should the trucks meet on a road to make them platooning. However, there are some cases that can make no-platooning is better than creating a platoon. This paper has been written in order to answer these questions and clarify these points.

In this contribution, this paper sheds light on whether and when trucks are advised to form a platoon. We help determine the optimal location for trucks to rendezvous in order to initiate a platoon. Lastly, we analyze the differences in arrival time for the trucks if they are using a platoon or using keep themselves isolated during their journey.

\section{Background}

There are ample research that target our subject and address various aspects that are considered in this paper.

Careful consideration is required on paths taken by trucks in order to determine whether there needs to be a platoon or not that platooning could impact at strategic and tactical level decisions as well [8]. The strategic level decisions may include physical design constructions [9]. These constructions could come in different ways such as adding new lanes for platoons. Recently, there are around 19 states such as Texas and Georgia are assigned a lane of their highways for platoons. Moreover, the roads status needs to take into consideration that they have ability for enduring heavy vehicles recurrently for instance reinforcement of roads and extra support in tunnels

One of the factors that supports the idea of platooning is reducing the fuel consumptions. Plenty of researches [10] [11] [12] show that the platooning can decrease the fuel consumptions up to $18 \%$. The saving fuel consumption goes back to aerodynamics specially on the following trucks that is increased when the distance between the trucks is reduced [13].

Speaking on the same approach, authors in [14] developed a formal model that can be applied in a platoon. The model can able to do some application on the platoon for instance merge and split. Merge operation can come in three types: tail merge, front merge, or could be a creation one if there are only two trucks need to be in one platoon. On the other side, spilt operation can be in three shapes such as a tail split, a front split, or could be a destroy split if and only if the platoon contains only two trucks. From the model in [14] we can get some benefits from platoon merge operations. Many studies have been performed on how and when vehicles should enter or leave platoons in traffic on or off-ramps [15], [16].

In the same direction of our talk, it would be better to mention how the impact of platooning size on roads. there is a study took a place in Netherland's roads [17] to see the impact of large-scale platooning on the road capacity by using a microscopic simulation model. The results were qualified whereas authors mentioned that platoon will increase the traffic flow by reducing the traffic time at the merging area in minimum intensity scenario for peak hour $1 \& 2$, and in minimum and mean scenario for the off-peak hour [17]. Moreover, the authors proved that the length of the platoon and its short inter-vehicle distance has a large impact on the merging behavior of cars but also on platoons 
themselves. Therefore, it can increase the chance of a moving bottleneck. In other words, platooning could be a great solution to break down traffic.

In the same context, another experiment was done by [18] to show the effect of platooning on roads efficiency. Authors in [18] expressed their idea in a simulation that shows the impact of platooning in light traffic, medium traffic and heavy traffic. The authors linked their work with the distance ratio that can help to reduce the fuel consumption that is mentioned in [19]. They proved that the merging distance increased directly proportional with traffic. However, the slower the lead HDV drives, the more difficult it is for the cars behind it to change lanes due to the big difference in speeds between the two lanes.

\section{Problem Statement}

Given a roadway map and a set of truck scattered on their paths from a common source to a common destination, our focus is on finding the best location to initiate platooning for the trucks. To be precise, the platooning point is meant to be the point that the trucks are willing to rendezvous in order to create a platoon for remainder of their travel to their destination. Frequently, the point could be a gas stations or a rest areas for instance. The platooning point could also be platoon stations- these kinds of stations which service platoon situations could be developed in the future. In addition, this paper discusses the best circumstance for trucks to be in platoons. The primary focus is on the arrival time for each truck. That is because the arrival time is one of the first factors that matters for successful freight delivery. Overall, we need to keep in mind the changeable weather, the road constructions status, as well as the roads that usually have congested traffic.

\section{Simulation Framework}

For the purpose of applying our idea for the platooning system, we employed prototyping software to conduct simulations. This is to avoid complexities of applying the idea on real trucks in the real world. We used AnyLogic 8.5 software that provides a platform the dynamic simulation modeling. We divided our simulation experiment into two parts: the three-truck part and the six-truck part. In the beginning, we started with the first part using three trucks from different locations heading to the same destination point with no platooning point as it is shown in fig. 1

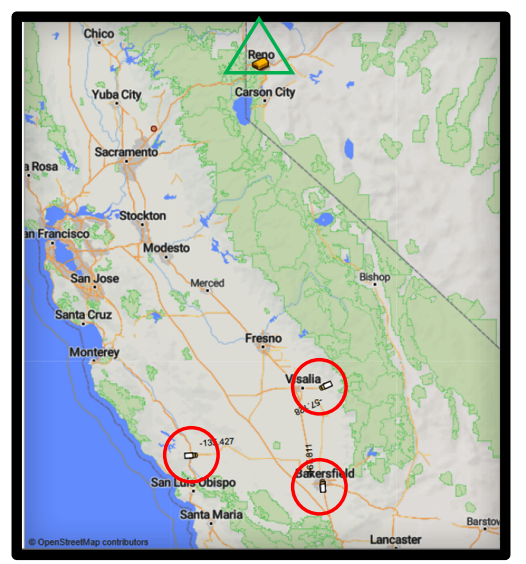

Figure 1: Three trucks "in the red circles", destination point "in the green tringle"-no platooning point 
We supposed that the three trucks can start from any location, at any time. However, all the three trucks are heading to one destination. That means, the three trucks may or may not using the same route. Nonetheless, there is a probability two out of three trucks may join in the same route whether at the same time or separately. We assumed all the three trucks are from one company. At the meantime, there is one manager can monitor all the trucks movement, who responsible to give the tasks to the trucks' drivers; the tasks could be: platoon, no platoon, indicate where is the platooning point in case of there is a platoon task. In the same regard,

each truck is implemented to now where other trucks are. At the same first part, we created another situation, three trucks as well but with a platooning point existed. In this situation, we tried the experiment around six times, each time with a different place of the platooning point in order to test where is the best result over all the situations In fig. 2.a, 2.b, 2.c, three different places for the platooning point in the three-truck part.

On the other hand, we designed the second part as follows: seven situations; one situation with no platooning point as it is displayed in fig. 3, and the other six situations included the platooning point. Each situation of these six-platooning situations has six trucks with a different place of the platooning point. The objective of having this part is to examine if we are planning to expand or increase the platoon size, does it make a better difference, or not? In the process of distribution of the platooning point, we took into consideration the distance between the platooning point and the trucks as well as the distance between the platooning point and the destination point.

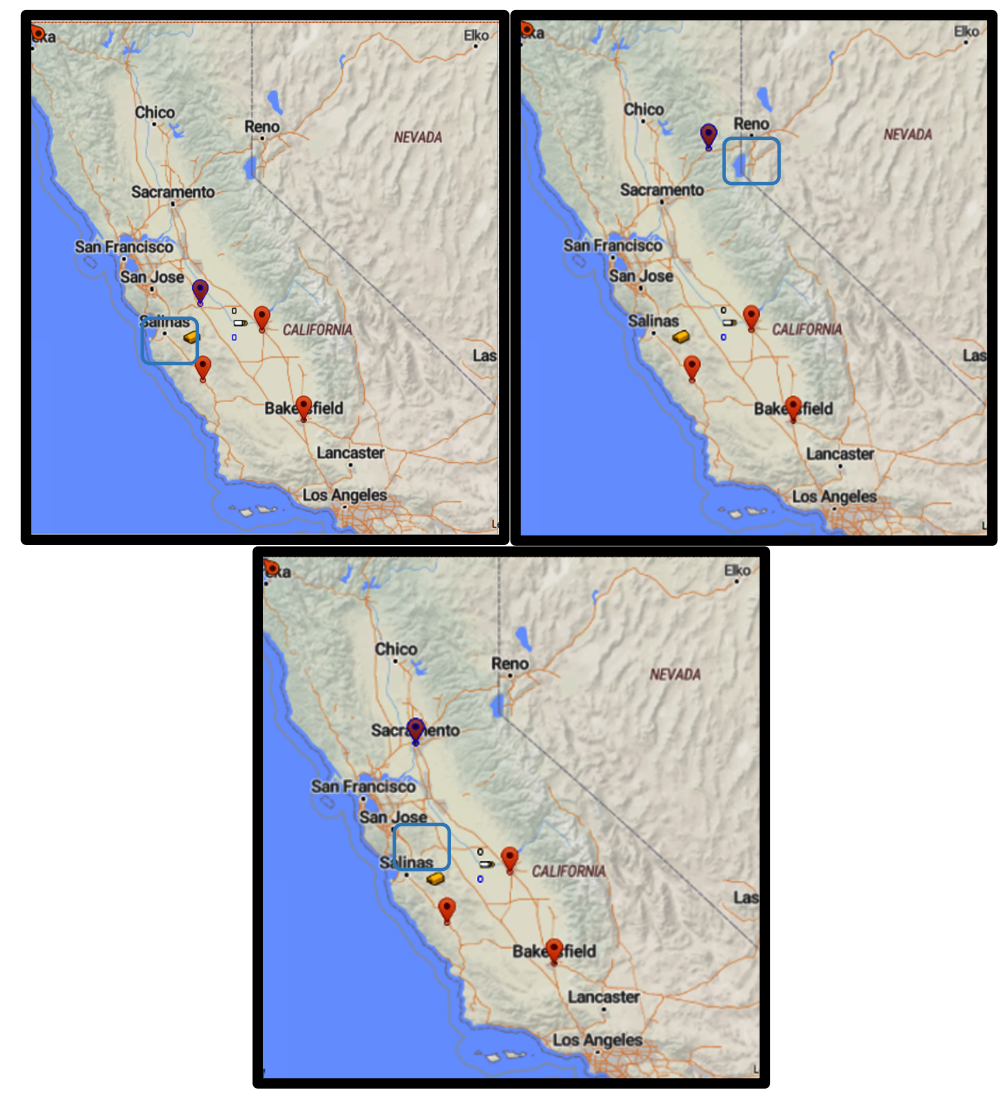

Figure 2.a, 2.b, 2.c: show the platooning point in different places with the blue rectangle 


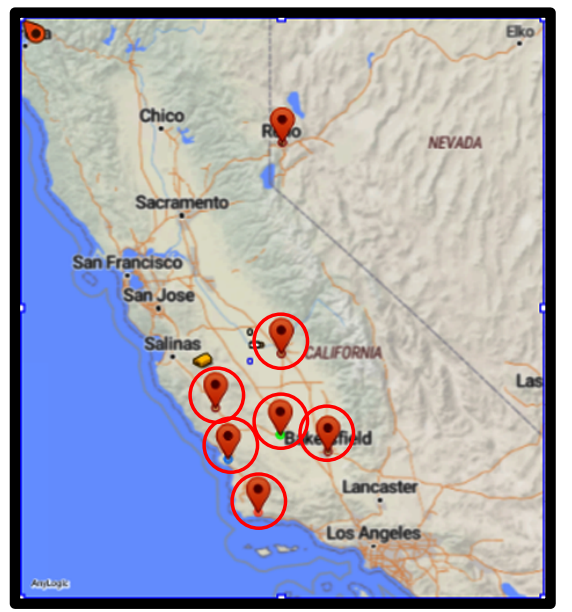

Figure 3: Six trucks with the red circles, destination point with the green tringle"-no platooning point

Put it differently, we created the platooning point sometimes closer to the trucks and farther from the destination point, and sometimes closer to the destination point, or sometimes in the middle. In fig. 4.a, 4.b, 4.c present some of the platooning point situations with six trucks.

When we ran the simulation, in the case if there is no platooning, all the trucks were heading to the destination point. In this case, we measured the time that each truck needs from its start point till the destination point. We set a constant value for the speed for each truck as $60 \mathrm{k} / \mathrm{m}$.

On the other side, the case with a platooning point, all the trucks were heading to the platooning point, and then waiting the other trucks that are planning to be platooned with. The time that the truck spending in the platooning point waiting other trucks is called: waiting time. Obviously, the waiting time for the last truck arriving to the platooning point is zero.

\section{Simulation Results}

In this section, we can pour what we got of results in a clear picture. To be precise, we are going to divide the results in two parts: three- truck platoon, and six-truck platoon. The three-truck part, its results showed that the first truck arrived to the destination in 5.2 hours with no platoon as it is shown in fig.5, while it arrived to the destination in 4.9 hours if it within a platoon in the first position of the platooning point in fig. 2.a. In the sense that the first truck arrived the destination point within a platoon with $5 \%$ time earlier which is better at least in fuel consumption. Whereas the second truck does not get much different arrival time among the three positions of the platooning point, but it is better than no platooning case. We can see the second truck arrived in 6.7 hours with no platoon, and it arrived in 6.3 hours in the first position of the platooning point in fig. 2.a. So do the same thing with the third truck; it arrived the destination point in 7.7 hours with no platoon while 7.0 hours within a platoon in the first position of the platooning point in Fig. 2a. 


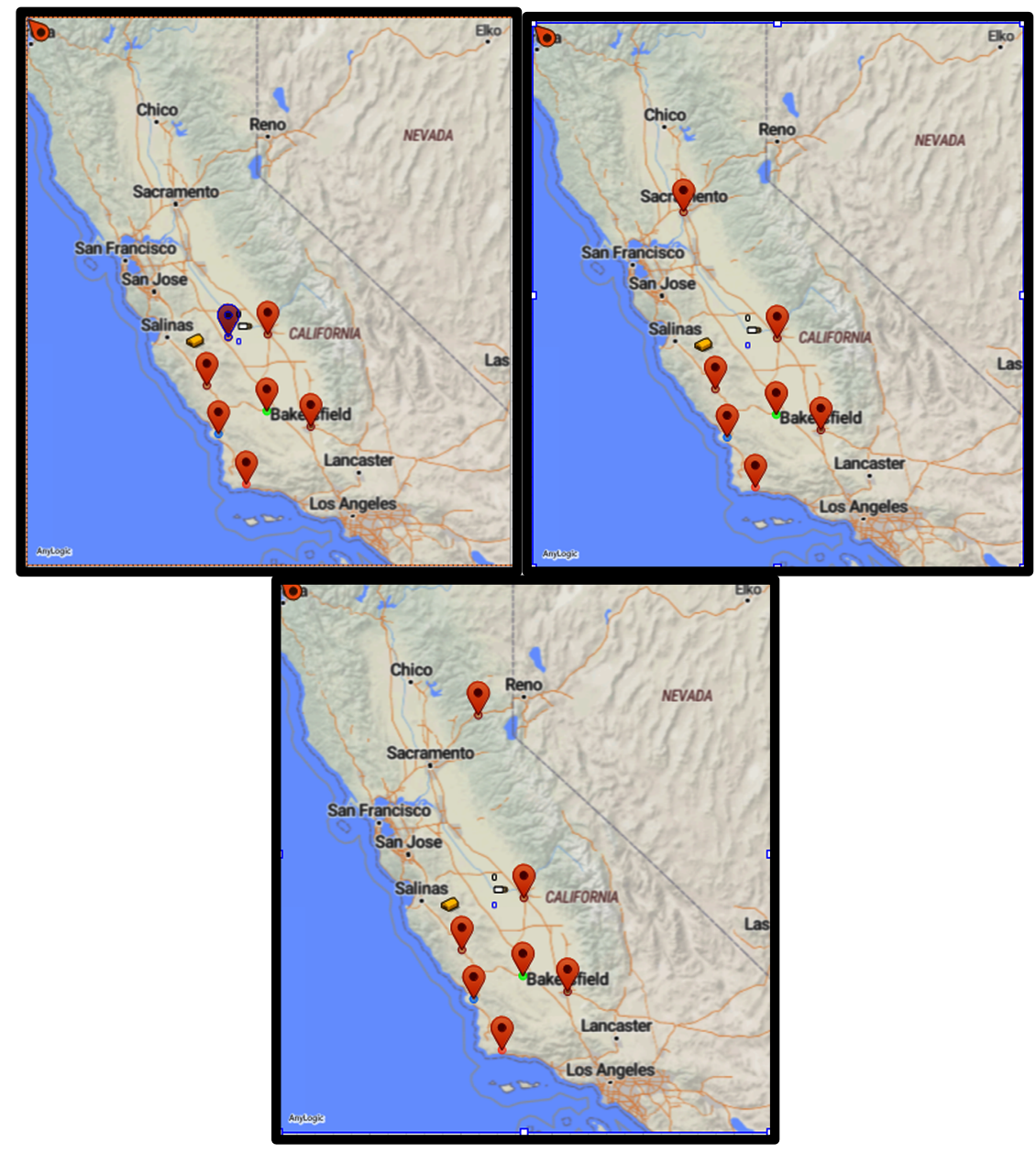

Figure 4.a, 4.b, 4.c: The platooning point in different places with the blue rectangle

Let us flip to the second part; the six-truck platoon. This part can be distinguished by saving more fuel, but at the same time the waiting time at the platooning point will be longer. For this reason, we should be careful with the arrival time for each truck as we are going to discuss this in the discussion section. We picked the most three obvious cases in this part and presented them in fig. 6 . It is apparent that the first position of platooning point that is shown in Fig. 4.a got the best arrival time results.

Although that the first truck arrival time in no platoon case and the second position of platooning point is better, but the other five trucks arrival time in the first position of platooning point are way better. Numerically, the arrival time of the first truck in the first position of platooning point is 5.5 hours comparing with 5.2 hours in no platoon case and the second position case; in the sense of nearly $5 \%$ difference. In contrast, the arrival time of the sixth truck in the first position of platooning point is 6.6 hours, and at the meantime, the arrival time of the sixth truck in no platoon case is 9.2 hours. Accordingly, the difference between these two cases are around 2.6 hours; in sense of nearly $28 \%$, which makes a better difference. 
Along the same lines, not only the sixth truck gets the benefit of platooning, but the other four trucks get the benefit as well. Whilst the second truck arrived the destination point in 6.7 hours in no platoon case, we can see the same truck arrived in 6.2 hours in the first position of platooning point. As well as the third truck that arrived in 7.6 hours in no platoon case comparing with 5.9 hours in the first position of platooning point. Third truck earned more benefits that the second truck, but both of these trucks are better than no platoon case. With the same status with the fourth truck that arrived in 6.1 hours in the first position of platooning point comparing with 7.3 hours in no platoon case. In addition, we can see there is a big difference between the arrival time of the fifth truck in the first position of platooning point that is 6.5 hours, and 8 hours in case of no platoon situation.

To conclude these, we can see some trucks got huge positive differences, and in contrast some of the other trucks got bit positive differences but it is still considered as good results if we considered fuel consumption side for instance.

\section{Discussion}

Under this section we will give an insight into on the gotten results. The main factor can affect on the arrival time between three-truck platoon and six-truck platoon is the waiting time in the platooning point. In other words, the first truck in three-truck platoon arrived the destination point in 4.9 hours at the first position of platooning point. However, at the same position of platooning point but with sixtruck platoon, the first truck arrived in 5.2 hours. The 0.3 of an hour difference is the waiting time the truck spent in the platooning point waiting five trucks arriving instead of waiting just two trucks in three-truck platoon case. To put it another way, the longer of platoon size we get, the longer waiting time we may have at the platooning point. Therefore, if we have longer size of platoon, we should perfectly make sure that the waiting time will have no effect on the arrival time of the trucks. Hence, if the waiting time at the platooning point does not affect the required arrival time at the destination point, then it is better to stick with the platoon.

The other point that should be considered is the fuel consumption. We can explicitly see that the relationship between the platooning-trip time and the fuel consumption is an inverse relationship.

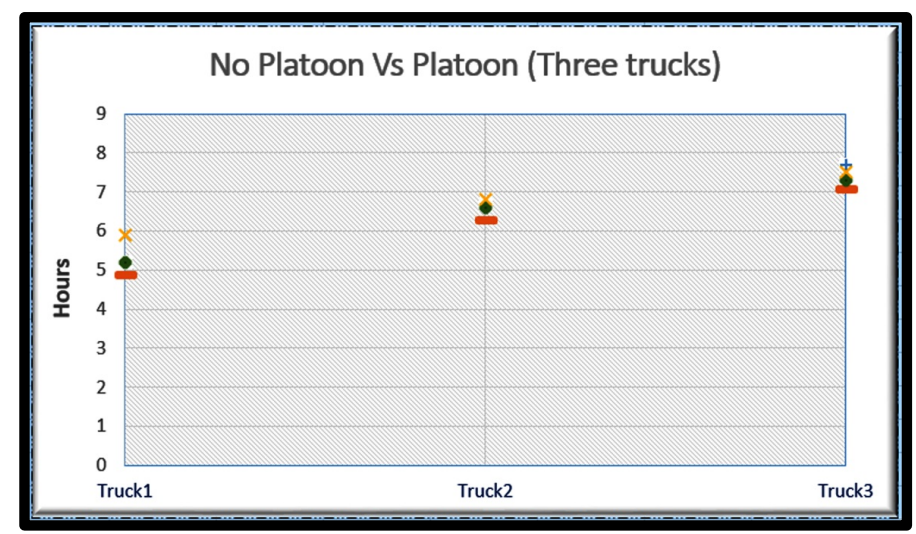

Figure 5: Three trucks arrival time (+ for no platoon, - the first position, $\bullet$ the second position, $\times$ the third position) 


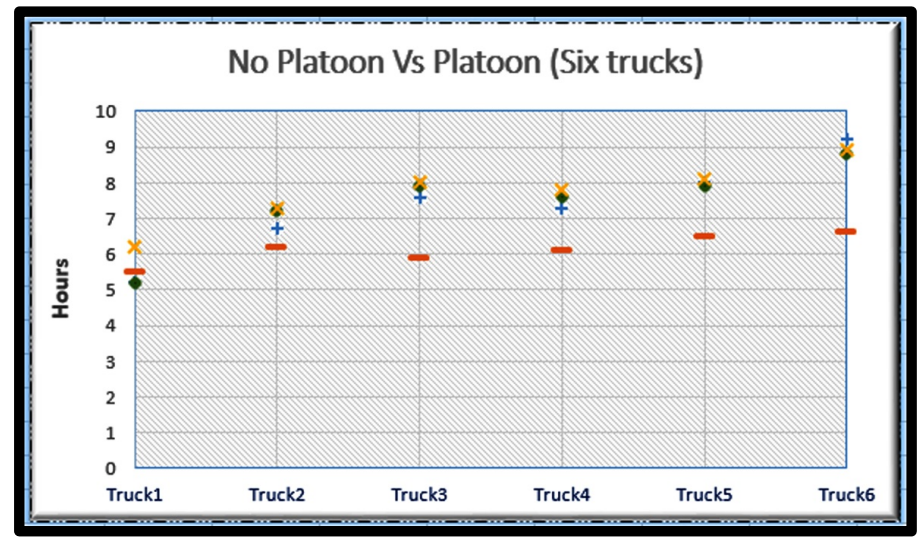

Figure 6: Six trucks arrival time (+ for no platoon, - the first position, $\downarrow$ the second position, $\times$ the third position)

That means, the longer time is spent for truck in platoon is less of fuel that is consumed. From this point, it is better to answer the significant question; where should be the platooning point? depending on all the experiments we have done, the platooning point place is better to be closer to the trucks position and farther from the destination point. For instance, if we take the first position of platooning point in the six-truck platoon, we can see that the platooning point is closer to the six trucks. To facilitate that, we drew a circle that can cover as many of the trucks as possible. Then, we picked the point on the circle and at the same time closer to the destination point, as it is shown in the fig. 7.

As our findings, the smaller circle we can make among the trucks, will give the shorter waiting time the trucks can have at the platooning point. Hence, the trucks will get shorter time to spend on the trip, and then the trucks will get earlier arrival time at the destination point. In other words, smaller circle we have, shorter waiting time the trucks will get at the platooning point.

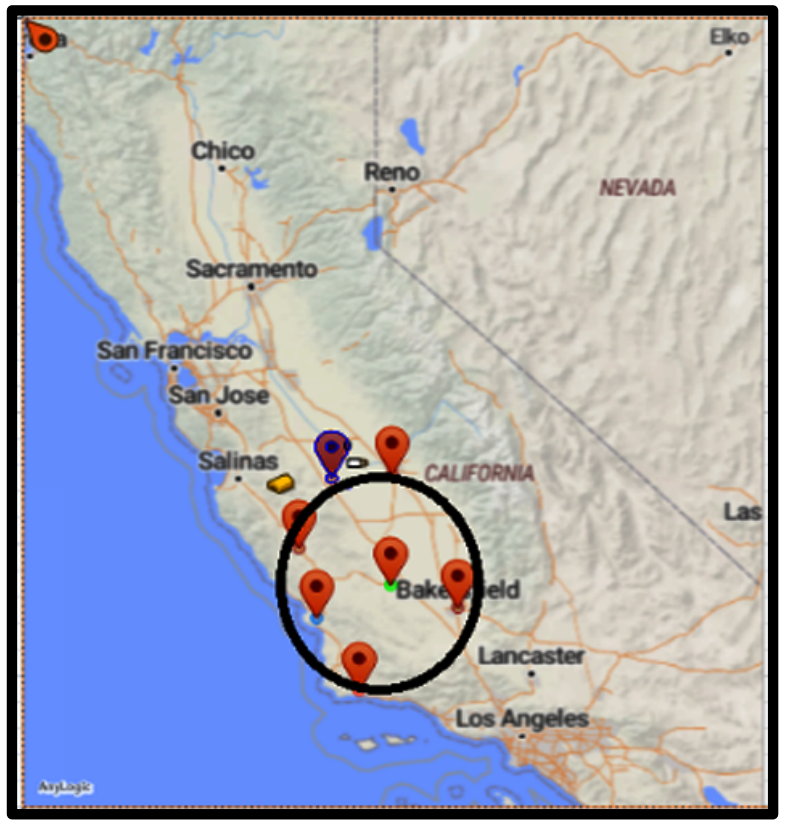

Figure 7: Circle shape among the trucks in order to help finding where is going to have the platooning point 


\section{Conclusions}

To recapitulate, the platoon system is a perfect solution when having multiple trucks heading to the same destination. Longer size of platooning is better when we shorter waiting time at the platooning point. On the contrary, if the trucks are far away from each other, it will give us a bigger circle among them. Hence, the trucks will have longer waiting time at the platooning point. So, in this case, it is better to split the trucks into groups depending on their locations in order to reduce the waiting time at the platooning point as the same case in the three-truck platoon. 


\section{References}

[1] R. Alshiddi, H. Hexmoor, "Path Finding and Joining for Truck Platoons," the 2019 International Conference on Artificial Intelligence, p. 1, 2019.

[2] C. Bonnet and H. Fritz, "Fuel consumption reduction in a platoon: Experimental results with two electronically coupled trucks at close spacing," SAE Technical Paper, p. 9, 2000.

[3] F. Browand, J. McArthur, and C. Radovich, "Fuel Saving Achieved in the Field Test of Two Tandem Trucks," Institute of Transportation Studies, Research Reports, Working Papers, Proceedings qt29v570mm, Institute of Transportation Studies, UC Berkeley, 2004.

[4] W. Zhang, M. Xiaoliang, J. Erik. "Planning of heavy-duty vehicle platoon formulation: basic scheduling (reem, 2000) problem considering travel time variance.” Transportation Research Board 95th Annual Meeting, 2016

[5] S. Tsugawa, S. Kato, and K. Aoki. "An automated truck platoon for energy saving.” In Intelligent Robots and Systems (IROS), 2011 IEEE/RSJ International Conference on, pages 4109-4114. IEEE, 2011

[6] T. Robinson, E. Chan, E. Coelingh, Operating Platoons On Public Motorways: An Introduction To The SARTRE Platooning Programme Proceedings of the 17th its world congress, Busan, Korea, October 25-29 2010.

[7] Vehicle Platooning Policy, Department of transportation in University of Pennsylvania-Department of transportation, 2019.

[8] A. K. Bhoopalam, N. Agatz, R. Zuidwijk, "Planning of Truck Platoons: a Literature Review and Directions for Future Research,” Transportation Research Part B: Methodological, Elsevier, vol. 107(C), pages 212-228, 2018.

[9] Z. Chen, F. He, Y. Yin, Y. Du, "Optimal design of autonomous vehicle zones in transportation networks," Transportation Research Part B: Methodological, Elsevier, vol. 99(C), pages 44-61, 2017.

[10] M. Roeth, "CR England Peloton Technology Platooning Test Nov 2013," in North American Council for Freight Efficiency, 2013, accessed: December 21, 2014. [Online]. Available: http://nacfe.org/wpcontent/uploads/2013/12/CR-England.pdf.

[11] X.-Y. Lu, S. E. Shladover, “Automated Truck Platoon Control and Field Test,” in Road Vehicle Automation, G. Meyer and S. Beiker, Eds. Springer International Publishing, 2014.

[12] S. van de Hoef, K. H. Johansson and D. V. Dimarogonas, "Fuel-Efficient En Route Formation of Truck Platoons," in IEEE Transactions on Intelligent Transportation Systems, vol. 19, no. 1, pp. 102-112, Jan. 2018.

[13] A. Alam, B. Besselink, V. Turri, J. Martensson, K. Johansson, "Heavy-duty vehicle platooning for sustainable freight transportation: A cooperative method to enhance safety and efficiency," IEEE Control Systems, 2015.

[14] S. Maiti, S. Winter, L. Kulik, "A conceptualization of vehicle platoons and platoon operations." Technologies and Control for Sustainable Transportation, 2017.

[15] L. D. Baskar, B. De Schutter, and H. Hellendoorn, "Dynamic speed limits and on-ramp metering for ivhs using model predictive control," in Proceedings of 11th International IEEE Conference on Intelligent Transportation Systems (ITSC), Beijing, China, 2008.

[16] V. Milan'es, J. Godoy, “Automated on-ramp merging system for congested traffic situations," IEEE Transactions on Intelligent Transportation Systems, vol. 12, no. 2, 2011.

[17] D. Yanga, A. Kuijpersa, G. Danea, T. Sande, "Impacts of large-scale truck platooning on Dutch highways," 21st EURO Working Group on Transportation Meeting, EWGT Transportation Research Procedia, 2018.

[18] K. Liang, Q. Deng, J. Mårtensson, X. Ma and K. H. Johansson, "The influence of traffic on heavy-duty vehicle platoon formation," 2015 IEEE Intelligent Vehicles Symposium (IV), Seoul, 2015, pp. 150-155.

[19] K. Liang, J. Martensson, and K. H. Johansson, "When is it Fuel Efficient for a Heavy Duty Vehicle to Catch Up With a Platoon?" in 7th IFAC Symposium on Advances in Automotive Control (IFAC-AAC), Tokyo, Japan, 2013 